\title{
Moderate Hypothermia to Treat Perinatal Asphyxial Encephalopathy
}

\author{
Denis V. Azzopardi, F.R.C.P.C.H., Brenda Strohm, R.G.N., \\ A. David Edwards, F.Med.Sci., Leigh Dyet, M.B., B.S., Ph.D., \\ Henry L. Halliday, F.R.C.P.H., Edmund Juszczak, M.Sc., \\ Olga Kapellou, M.D., Malcolm Levene, F.Med.Sci., Neil Marlow, F.Med.Sci., \\ Emma Porter, M.R.C.P.C.H., Marianne Thoresen, M.D., Ph.D., \\ Andrew Whitelaw, F.R.C.P.C.H., and Peter Brocklehurst, F.F.P.H., \\ for the TOBY Study Group*
}

A BSTRACT

BACKGROUND

Whether hypothermic therapy improves neurodevelopmental outcomes in newborn infants with asphyxial encephalopathy is uncertain.

\section{METHODS}

We performed a randomized trial of infants who were less than 6 hours of age and had a gestational age of at least 36 weeks and perinatal asphyxial encephalopathy. We compared intensive care plus cooling of the body to $33.5^{\circ} \mathrm{C}$ for 72 hours and intensive care alone. The primary outcome was death or severe disability at 18 months of age. Prespecified secondary outcomes included 12 neurologic outcomes and 14 other adverse outcomes.

\section{RESULTS}

Of 325 infants enrolled, 163 underwent intensive care with cooling, and 162 underwent intensive care alone. In the cooled group, 42 infants died and 32 survived but had severe neurodevelopmental disability, whereas in the noncooled group, 44 infants died and 42 had severe disability (relative risk for either outcome, 0.86; 95\% confidence interval [CI], 0.68 to $1.07 ; \mathrm{P}=0.17)$. Infants in the cooled group had an increased rate of survival without neurologic abnormality (relative risk, 1.57; 95\% CI, 1.16 to 2.12; $\mathrm{P}=0.003)$. Among survivors, cooling resulted in reduced risks of cerebral palsy (relative risk, $0.67 ; 95 \% \mathrm{CI}, 0.47$ to $0.96 ; \mathrm{P}=0.03$ ) and improved scores on the Mental Developmental Index and Psychomotor Developmental Index of the Bayley Scales of Infant Development II ( $\mathrm{P}=0.03$ for each) and the Gross Motor Function Classification System $(\mathrm{P}=0.01)$. Improvements in other neurologic outcomes in the cooled group were not significant. Adverse events were mostly minor and not associated with cooling.

\section{CONCLUSIONS}

Induction of moderate hypothermia for 72 hours in infants who had perinatal asphyxia did not significantly reduce the combined rate of death or severe disability but resulted in improved neurologic outcomes in survivors. (Current Controlled Trials number, ISRCTN89547571.)

From the Division of Clinical Sciences and Medical Research Council (MRC) Clinical Sciences Centre, Hammersmith Hospital, Imperial College London, London (D.V.A., A.D.E., L.D., O.K., E.P.); the National Perinatal Epidemiology Unit, University of Oxford, Oxford (B.S., E.J., P.B.); the Department of Perinatal Medicine, Royal Maternity Hospital and Department of Child Health, Queen's University, Belfast (H.L.H.); the University of Leeds and Leeds General Infirmary, Leeds (M.L.); the Academic Division of Child Health, Queen's Medical Centre, Nottingham (N.M.); and the Department of Clinical Science, University of Bristol, St. Michael's Hospital (M.T.) and Southmead Hospital (A.W.), Bristol - all in the United Kingdom. Address reprint requests to Dr. Azzopardi at the Division of Clinical Sciences and MRC Clinical Sciences Centre, Imperial College London, Hammersmith Hospital, Du Cane Rd., London W12 ONN, United Kingdom, or at d.azzopardi@ imperial.ac.uk.

\footnotetext{
*The members of the Total Body Hypothermia for Neonatal Encephalopathy Trial (TOBY) study group are listed in the Appendix.
}

N EnglJ Med 2009;361:1349-58

Copyright (๑) 2009 Massachusetts Medical Society. 
P ERINATAL ASPHYXIAL ENCEPHALOPATHY is associated with high morbidity and mortality rates worldwide and is a major burden for the patient, the family, and society. There is an urgent need to improve outcomes in affected infants.

Experimentally, reducing body temperature to 3 to $5^{\circ} \mathrm{C}$ below the normal level reduces cerebral injury and improves neurologic function after asphyxia. ${ }^{1-6}$ Preliminary clinical studies have found no serious adverse effects of cooling. ${ }^{7-9}$ Two randomized, controlled trials, the CoolCap trial ${ }^{10}$ and the National Institute of Child Health and $\mathrm{Hu}$ man Development (NICHD) trial, ${ }^{11}$ have reported outcomes among infants at 18 months of age who had asphyxial encephalopathy, after slightly different cooling regimens. Only the NICHD trial showed a significant reduction in the composite primary outcome of death or disability with hypothermia. Neither trial had sufficient power to detect significant differences in important individual neurologic outcomes, and several systematic reviews and an expert workshop did not reach a consensus in recommending hypothermia as standard treatment. ${ }^{12-17}$

To clarify the role of hypothermia, we carried out the Total Body Hypothermia for Neonatal Encephalopathy Trial (TOBY), a multicenter, randomized trial comparing intensive care plus total-body cooling for 72 hours with intensive care without cooling among term infants with asphyxial encephalopathy.

\section{METHODS}

The TOBY protocol was approved by the London Multicenter Research Ethics Committee and the local research ethics committee of each participating hospital. Conduct of the study was overseen by an independent trial steering committee with advice from an independent data monitoring and ethics committee.

\section{STUDY DESIGN AND PROCEDURES}

TOBY was a randomized, controlled trial, involving term infants, comparing intensive care plus total-body cooling for 72 hours with intensive care without cooling. Infants were eligible if they were born at or after 36 completed weeks' gestation. They also had to have, at 10 minutes after birth, either an Apgar score of 5 or less or a continued need for resuscitation or, within 60 minutes after birth, acidosis (defined as any occurrence of umbilical-cord, arterial, or capillary $\mathrm{pH}$ of $<7.00$ or base deficit of $\geq 16 \mathrm{mmol}$ per liter). In addition, they had to have moderate-to-severe encephalopathy (indicated by lethargy, stupor, or coma) and either hypotonia, abnormal reflexes (including oculomotor or pupillary abnormalities), an absent or weak suck, or clinical seizures. Finally, they had to have abnormal background activity of at least 30 minutes' duration or seizures on amplitudeintegrated electroencephalography. ${ }^{18}$

We excluded infants expected to be more than 6 hours of age at the time of randomization and those with major congenital abnormalities known at randomization that required surgery or were suggestive of chromosomal anomaly or syndromes that involve brain dysgenesis.

Written informed consent was obtained from a parent of each infant after explanation of the study, and consent was reaffirmed within the subsequent 24 hours. ${ }^{19}$ Assignment to a treatment group was performed by means of central telephone randomization or a secure Web-based system (provided by the National Perinatal Epidemiology Unit Clinical Trials Unit, Oxford, United Kingdom). Minimization was used to ensure balance of treatment assignment among infants with various grades of abnormality on amplitude-integrated electroencephalography and within each participating center.

\section{CLINICAL MANAGEMENT}

All recruited infants were cared for in participating centers. Infants from referring hospitals were assessed by trained retrieval teams who performed amplitude-integrated electroencephalography, sought consent if the infant was eligible, performed randomization, and for infants assigned to the cooled group, began cooling by discontinuing warming and applying cooled gel packs, if necessary, until the infant was admitted to a participating center.

To minimize potential confounding from differential use of cointerventions, uniform guidance was provided on ventilatory and circulatory care, management of seizures, sedation, and fluid requirements. All infants underwent sedation with morphine infusions or with chloral hydrate if they appeared to be distressed. Skin temperature and rectal temperature (measured at least $2 \mathrm{~cm}$ within the rectum) were monitored continuously and recorded hourly in all infants throughout the inter- 
vention period. Clinical staff were made aware of the treatment assignments so that they could manage cooling appropriately.

\section{Intensive Care Alone}

Infants assigned to the noncooled group received the current standard of care and were placed under radiant heaters or in incubators, which were servo-controlled according to the abdominal skin temperature to maintain the rectal temperature at $37.0 \pm 0.2^{\circ} \mathrm{C}$.

\section{Intensive Care with Cooling}

Infants assigned to the cooled group were treated in incubators with the power turned off. Hypothermia was maintained by nursing the infant on a cooling blanket in which fluid whose temperature was regulated by a manually adjusted thermostat (Tecotherm TS 200, Tec-Com) was circulated. The target rectal temperature was 33 to $34^{\circ} \mathrm{C}$, and typically, the thermostat was set from 25 to $30^{\circ} \mathrm{C}$.

\section{Rewarming Procedures}

When the period of cooling concluded, 72 hours after randomization, the rectal temperature was monitored for at least 4 hours to prevent rebound hyperthermia. The rectal temperature was allowed to rise by no more than $0.5^{\circ} \mathrm{C}$ per hour, to a maximum of $37 \pm 0.2^{\circ} \mathrm{C}$. Cranial ultrasonography was performed daily for the first 4 days after birth, and magnetic resonance imaging (MRI) was conducted, according to a specified protocol, within 5 to 14 days after birth.

\section{OUTCOMES}

The primary outcome at 18 months of age was a composite of death or severe neurodevelopmental disability in survivors. Severe neurodevelopmental disability was defined as a score of less than 70 on the Mental Developmental Index of the Bayley Scales of Infant Development II (BSID-II) (on which the standardization mean $[ \pm S D]$ is $100 \pm 15$ and higher scores indicate better performance), a score of 3 to 5 on the Gross Motor Function Classification System ${ }^{20}$ (GMFCS) (on which scores can range from 1 to 5 , with higher scores indicating greater impairment), or bilateral cortical visual impairment with no useful vision.

Adverse outcomes included intracranial hemorrhage, persistent hypotension, pulmonary hemorrhage, pulmonary hypertension, prolonged blood coagulation time, culture-proven sepsis, necrotizing enterocolitis, cardiac arrhythmia, thrombocy- topenia, major venous thrombosis, renal failure treated with dialysis, pneumonia, pulmonary air leak, and duration of hospitalization. (Most outcomes are defined in the Supplementary Appendix, available with the full text of this article at NEJM.org.)

Secondary outcomes at 18 months, specified before data analysis, included death and severe neurodevelopmental disability (components of the composite primary outcome), the score on the Psychomotor Developmental Index of BSID-II (on which the standardization mean $[ \pm S D]$ is $100 \pm 15$ and higher scores indicate better performance), cerebral palsy, hearing loss, seizures treated with anticonvulsant agents, microcephaly (i.e., age- and sex-standardized head circumference of more than 2 SD below the mean), multiple neurodevelopmental abnormalities (i.e., more than one of the following: a GMFCS score of 3 to 5 , a score of $<70$ on the Mental Developmental Index of BSID-II) (on which the standardization mean $[ \pm S D]$ is $100 \pm 15$ and higher scores indicate better performance, seizures, or cortical visual impairment and hearing loss), and survival without neurologic abnormality (i.e., a Mental Developmental Index score $>84$, a Psychomotor Developmental Index score $>84$, no abnormalities on GMFCS assessment, and normal vision and hearing).

\section{NEUROLOGIC ASSESSMENT}

Infants were assessed at approximately 18 months of age, through a structured examination by one of five trained assessors who were unaware of the treatment assignments. Neurologic signs and function were scored, ${ }^{20,21}$ and the presence and type of cerebral palsy were determined. Neurodevelopmental outcome was assessed according to the BSID-II. ${ }^{22}$

\section{STATISTICAL ANALYSIS}

We estimated that a sample of 236 infants would be required to detect a relative risk of 0.6 to 0.7 for the primary outcome in the cooled group as compared with the noncooled group, with a statistical power of $80 \%$, at a two-sided significance level of $5 \%$ and assuming a $10 \%$ loss to follow-up. This sample size was achieved ahead of schedule, and enrollment was continued after the CoolCap and NICHD trial results suggested that a larger sample would be valuable.

Demographic and clinical characteristics were summarized at baseline as counts and percentages of the total numbers of infants for categorical vari- 


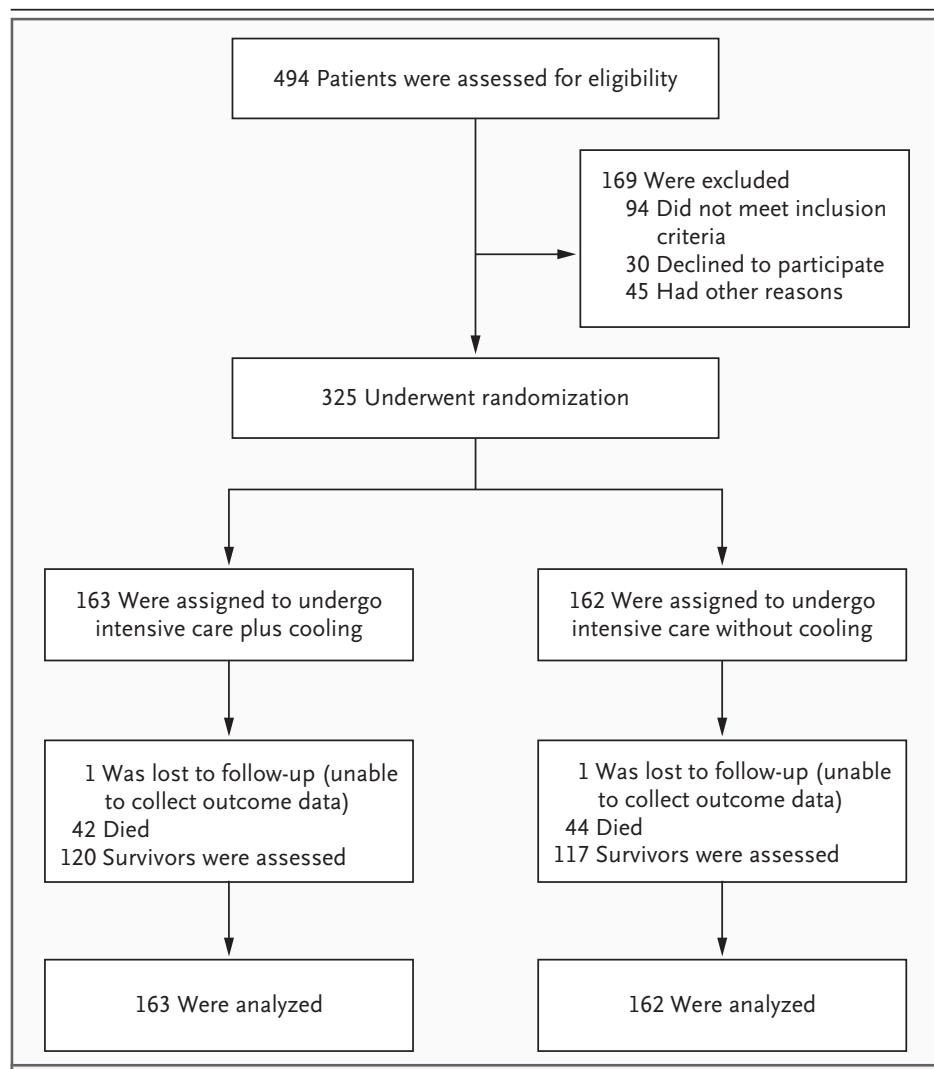

Figure 1. Enrollment and Follow-up of the Study Infants. with stratification on the basis of the grade of abnormality on amplitude-integrated electroencephalography at randomization and duration of the interval between birth and randomization ( 0 to $<4$ hours vs. 4 to 6 hours). The consistency of the effect of the treatment group across subgroups was explored by means of the statistical test of interaction.

\section{RESULTS}

From December 1, 2002, through November 30, 2006, 494 infants were screened and 325 were recruited from 42 hospitals (Fig. 1). The infants were from the United Kingdom (277), Hungary (24), Sweden (18), Israel (4), and Finland (2). Baseline characteristics of the infants (including maternal characteristics) were broadly similar between the two groups (Table 1).

\section{COMPLIANCE WITH COOLING PROTOCOL}

Rectal temperatures were similar between the two groups at the time of randomization (Table 1 ). Mean rectal temperatures at 6 to 72 hours after randomization were $33.5 \pm 0.5^{\circ} \mathrm{C}$ and $36.9 \pm 0.6^{\circ} \mathrm{C}$ in the cooled and noncooled groups, respectively (Fig. 2). Among the 162 infants who were not cooled, during the treatment period the temperature rose above $38^{\circ} \mathrm{C}$ on one occasion in $14(9 \%)$ and on more than one occasion in 23 (14\%). continuous variables, and as medians and ranges or interquartile ranges for other continuous variables.

Data were analyzed in the groups to which patients had been assigned regardless of either deviation from the protocol or treatment received. Consistent with previous reports, ${ }^{10,11}$ neurologic outcomes are presented for survivors who had available follow-up data.

Comparative statistical analysis entailed calculating the relative risks plus the $95 \%$ confidence intervals for all dichotomous outcomes, the mean differences plus $95 \%$ confidence intervals for normally distributed, continuous outcomes (using analysis of covariance where appropriate), and the median differences plus $95 \%$ confidence intervals for skewed continuous variables. In addition, ordered categorical variables were examined with the use of the chi-square test for trend. An adjusted analysis of the primary outcome was performed to investigate the effect of known prognostic factors. All statistical tests were two-sided and were not adjusted for multiple comparisons.

Prespecified subgroup analyses were performed

\section{PRIMARY OUTCOME}

In the cooled group, 42 infants died and 32 survived with severe neurodevelopmental disability, whereas in the noncooled group, 44 infants died and 42 had severe disability (Table 2) (relative risk for either outcome, 0.86 ; $95 \%$ confidence interval [CI], 0.68 to $1.07 ; \mathrm{P}=0.17)$. The result was materially unchanged when adjusted for severity of abnormality on amplitude-integrated electroencephalography, sex, or age at randomization.

\section{ADVERSE OUTCOMES}

The incidence of adverse events was similar in the two groups (Table 3). Hypotension, thrombocytopenia, prolonged coagulation time, and intracranial hemorrhage (seen only on MRI) were frequently observed in both groups. Serious adverse events other than death were uncommon and were not associated with cooling. Two infants in the cooled group and one in the noncooled group had sinus thrombosis noted on MRI. Another infant in the cooled group had a thrombus in the aorta, 


\begin{tabular}{|c|c|c|c|}
\hline Characteristic & $\begin{array}{l}\text { Cooled Group } \\
(\mathrm{N}=163)\end{array}$ & $\begin{array}{l}\text { Noncooled Group } \\
(\mathrm{N}=162)\end{array}$ & P Value \\
\hline Male sex — no. (\%) & $101(62)$ & $88(54)$ & 0.16 \\
\hline \multicolumn{4}{|l|}{ Gestational age - wk } \\
\hline Median & 40.3 & 40.1 & 0.22 \\
\hline IQR & $39.1-41.3$ & $38.8-41.1$ & \\
\hline \multicolumn{4}{|l|}{ Birth weight $-\mathrm{g}$} \\
\hline Median & 3450 & 3350 & 0.18 \\
\hline IQR & $2957-3873$ & $3044-3729$ & \\
\hline \multicolumn{4}{|l|}{ Head circumference $-\mathrm{cm}$} \\
\hline Median & 35.0 & 35.0 & 0.53 \\
\hline IQR & $34.0-36.0$ & $34.0-35.9$ & \\
\hline \multicolumn{4}{|l|}{ Age at randomization } \\
\hline Median - hr & 4.7 & 4.7 & 0.88 \\
\hline $\mathrm{IQR}-\mathrm{hr}$ & $3.8-5.4$ & $3.5-5.5$ & \\
\hline 0 to $<4 \mathrm{hr}-$ no. (\%) & $48(29)$ & $57(35)$ & 0.27 \\
\hline 4 to $6 \mathrm{hr}-$ no. (\%) & $115(71)$ & $105(65)$ & \\
\hline Maternal pyrexia during labor — no. (\%) $†$ & $10(6)$ & $10(6)$ & 0.94 \\
\hline Delivery complications — no. (\%) & $115(71)$ & $119(74)$ & 0.55 \\
\hline \multicolumn{4}{|l|}{ Apgar score at $10 \mathrm{~min}$} \\
\hline Median & 4 & 4 & 0.15 \\
\hline IQR & $2-5$ & $2-5$ & \\
\hline$<5-$ no. $(\%)$ & $110(83)$ & $105(77)$ & 0.21 \\
\hline Resuscitation required at $10 \mathrm{~min}$ of age - no. (\%) & $149(91)$ & $151(93)$ & 0.54 \\
\hline Clinical seizures — no. (\%) & $92(56)$ & $83(51)$ & 0.35 \\
\hline \multicolumn{4}{|l|}{ Temperature at randomization } \\
\hline Mean $\pm \mathrm{SD}-{ }^{\circ} \mathrm{C}$ & $36.6 \pm 1.1$ & $36.5 \pm 1.2$ & 0.24 \\
\hline$<35.5^{\circ} \mathrm{C}-$ no. $(\%)$ & $18(11)$ & $25(16)$ & 0.24 \\
\hline \multicolumn{4}{|l|}{ Abnormality on aEEG at randomization - no. (\%) } \\
\hline Moderate & $65(40)$ & $67(41)$ & \\
\hline Severe & $98(60)$ & $95(59)$ & 0.79 \\
\hline
\end{tabular}

* Data were unavailable for some patients: for gestational age, 16 patients in the cooled group and 17 in the noncooled group; for birth weight, 1 patient in the cooled group and 1 in the noncooled group; for head circumference, 41 patients in the cooled group and 44 in the noncooled group; for maternal pyrexia, 2 patients in the cooled group and 6 in the noncooled group; for delivery complications, 2 patients in the cooled group and 2 in the noncooled group; for Apgar score at 10 minutes, 31 patients in the cooled group and 26 in the noncooled group; and for temperature at randomization, 2 patients in the noncooled group. The term aEEG denotes amplitude-integrated electroencephalography, and IQR interquartile range.

$\uparrow$ Pyrexia was defined as a temperature of $37.6^{\circ} \mathrm{C}$ or more.

as well as an umbilical arterial catheter and a hematocrit of $70 \%$. No case of renal failure requiring dialysis occurred.

\section{SECONDARY OUTCOMES AT 18 MONTHS}

The mortality rate was similar in both groups. Forty-two infants in the cooled group died, as did 44 infants in the noncooled group; in each group, 39 of those died before hospital discharge. Death occurred after the withdrawal of care in 34 of the $39(87 \%)$ in the cooled group and 29 of the 39 (74\%) in the noncooled group.

Outcomes were significantly improved in the cooled group with regard to 5 of the 12 secondary neurologic outcomes assessed (Table 2). The rate of survival without a neurologic abnormality was significantly increased in the cooled group (71 of 163 infants [44\%], vs. 45 of 162 [28\%] in 


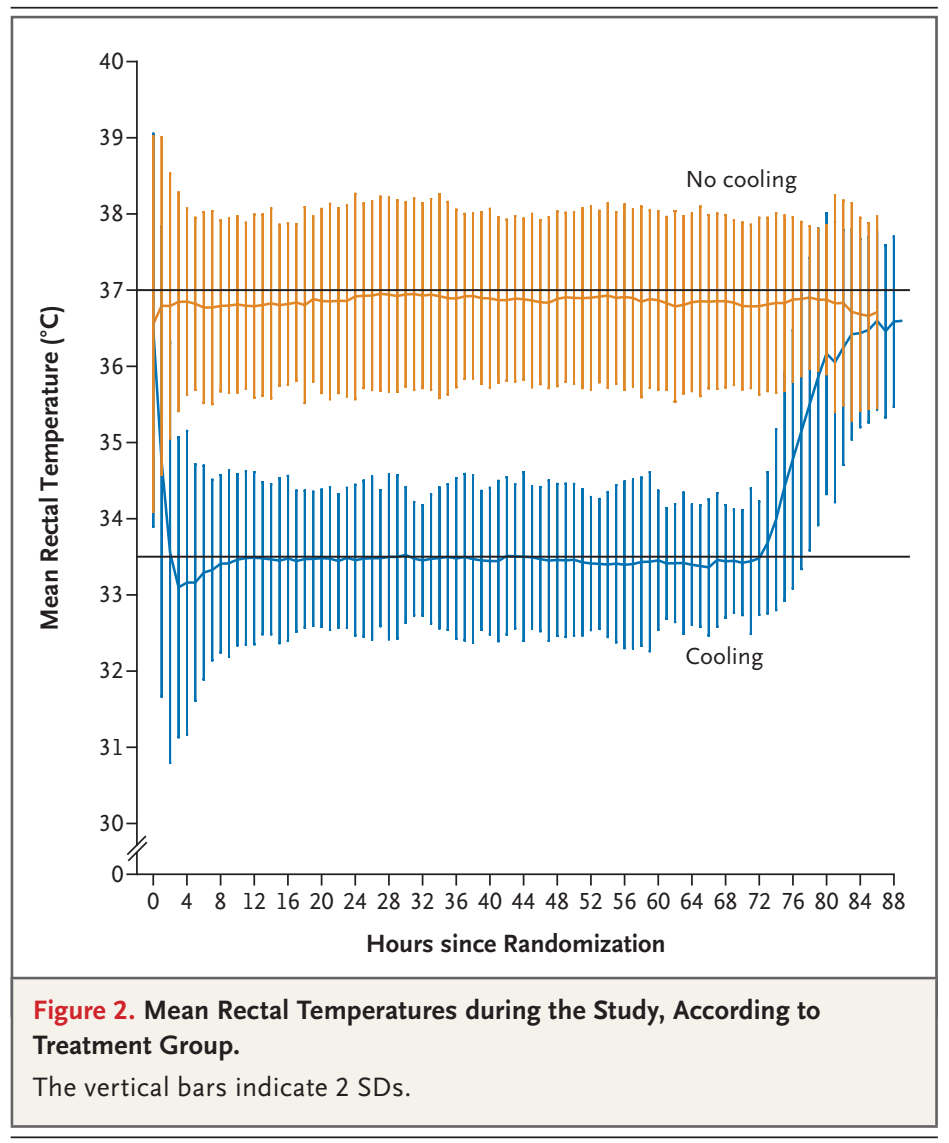

the noncooled group; relative risk, 1.57; 95\% CI, 1.16 to $2.12 ; \mathrm{P}=0.003$ ). Among survivors, cooling resulted in reduced risks of cerebral palsy (relative risk, $0.67 ; 95 \% \mathrm{CI}, 0.47$ to $0.96 ; \mathrm{P}=0.03$ ) and abnormal GMFCS score (relative risk, 0.63 ; 95\% CI, 0.45 to $0.89 ; \mathrm{P}=0.007$ ) and resulted in improvements in the Mental Developmental Index and Psychomotor Developmental Index scores $(\mathrm{P}=0.03$ for each), and the GMFCS score ( $\mathrm{P}=0.01)$. The rate of multiple neurodevelopmental abnormalities was 21 of 112 in the cooled group, as compared with 33 of 110 in the noncooled group (relative risk, $0.63 ; 95 \% \mathrm{CI}, 0.39$ to $1.01 ; \mathrm{P}=0.05$ ). Results from the complete analysis of the neurodevelopmental assessments are provided in the Supplementary Appendix.

\section{SUBGROUP ANALYSES}

More infants with severely abnormal results on amplitude-integrated electroencephalography at the time of randomization died or had a severe disability than did those with moderately abnormal results (109 of 193 [56\%] vs. 50 of 132 [38\%]; relative risk, $1.49 ; 95 \% \mathrm{CI}, 1.16$ to $1.91 ; \mathrm{P}=0.001$ ).
However, the effect of cooling did not significantly vary according to the severity of abnormality on amplitude-integrated electroencephalography ( $\mathrm{P}=0.23$ for interaction). The results were similar when the analysis was carried out with the results of amplitude-integrated electroencephalography classified as in the CoolCap study. ${ }^{10}$ The effect of treatment group did not vary significantly on the basis of time to randomization: among the 105 infants randomly assigned to a group less than 4 hours after birth, the relative risk for the primary outcome with cooling was 0.77 ( $95 \%$ CI, 0.44 to 1.04), whereas among the 220 remaining infants who were randomly assigned between 4 and 6 hours after birth, the relative risk was 0.95 ( $95 \% \mathrm{CI}, 0.72$ to $1.25 ; \mathrm{P}=0.21$ for interaction).

\section{DISCUSSION}

In this trial of near-term infants with perinatal asphyxia, we found no significant difference in the risk of the primary outcome, the combined rates of death or severe disability, between the cooled group and the noncooled group. However, cooling resulted in consistent improvement in secondary outcomes, including a significant increase in the rate of survival without neurologic abnormalities and improved neurodevelopmental outcomes among survivors.

The primary outcome of TOBY, as in the CoolCap and NICHD trials, was a composite end point, chosen because of concerns that cooling might increase survival with additional disability. Results of all three trials are consistent with respect to this primary outcome, with point estimates supporting a benefit from hypothermia: the relative risk associated with cooling (vs. no cooling) was 0.82 (95\% CI, 0.66 to 1.02) in the CoolCap study, 0.72 (95\% CI, 0.71 to 0.93 ) in the NICHD trial (which included infants with moderate disability), and 0.86 (95\% CI, 0.68 to 1.07 ) in the present trial.

Our categorization of neurologic outcomes is consistent with that used in the CoolCap and NICHD trials and previous systematic reviews, ${ }^{12-17}$ facilitating the comparison of our findings with previous results. We found a significant increase in the rate of survival without neurologic abnormality with cooling (relative risk, 1.57; 95\% CI, 1.16 to 2.12); the NICHD and CoolCap trials, which were smaller than the present trial, showed nonsignificant benefits with regard to this out- 


\begin{tabular}{|c|c|c|c|c|}
\hline \multirow[t]{2}{*}{ Outcome } & Cooled Group & Noncooled Group & P Value & $\begin{array}{l}\text { Relative Risk } \\
(95 \% \mathrm{CI})\end{array}$ \\
\hline & \multicolumn{2}{|c|}{ no./total no. (\%) } & & \\
\hline \multicolumn{5}{|l|}{ Primary outcome } \\
\hline $\begin{array}{l}\text { Combined death and severe } \\
\text { neurodevelopmental disability }\end{array}$ & $74 / 163(45)$ & $86 / 162(53)$ & 0.17 & $0.86(0.68-1.07)$ \\
\hline \multicolumn{5}{|l|}{ Secondary outcomes* } \\
\hline Death & $42 / 163(26)$ & $44 / 162(27)$ & 0.78 & $0.95(0.66-1.36)$ \\
\hline Severe neurodevelopmental disability & $32 / 120(27)$ & $42 / 117(36)$ & 0.13 & $0.74(0.51-1.09)$ \\
\hline Survival without neurologic abnormality & $71 / 163(44)$ & $45 / 162(28)$ & 0.003 & $1.57(1.16-2.12)$ \\
\hline Multiple neurodevelopmental disabilities & 21/112 (19) & $33 / 110(30)$ & 0.05 & $0.63(0.39-1.01)$ \\
\hline BSID-II Mental Developmental Index score & & \multicolumn{3}{|c|}{0.03 for trend } \\
\hline$<70$ & $28 / 115(24)$ & $38 / 110(35)$ & 0.09 & $0.70(0.47-1.06)$ \\
\hline $70-84$ & $6 / 115(5)$ & $12 / 110(11)$ & & \\
\hline$\geq 85$ & $81 / 115(70)$ & $60 / 110(55)$ & 0.01 & $1.29(1.05-1.59)$ \\
\hline $\begin{array}{l}\text { BSID-II Psychomotor Developmental Index } \\
\text { score }\end{array}$ & & \multicolumn{3}{|c|}{0.03 for trend } \\
\hline$<70$ & $27 / 114(24)$ & $37 / 109(34)$ & 0.09 & $0.70(0.46-1.06)$ \\
\hline $70-84$ & $9 / 114(8)$ & $14 / 109(13)$ & & \\
\hline$\geq 85$ & $78 / 114(68)$ & $58 / 109(53)$ & 0.02 & $1.29(1.04-1.60)$ \\
\hline GMFCS score & & \multicolumn{3}{|c|}{0.01 for trend } \\
\hline No abnormality & $85 / 120(71)$ & $63 / 117(54)$ & 0.007 & $1.32(1.07-1.61)$ \\
\hline $1-2$ & $11 / 120(9)$ & $18 / 117(15)$ & & \\
\hline $3-5$ & $24 / 120(20)$ & $36 / 117(31)$ & 0.06 & $0.65(0.41-1.02)$ \\
\hline Cerebral palsy & $33 / 120(28)$ & $48 / 117(41)$ & 0.03 & $0.67(0.47-0.96)$ \\
\hline Hearing loss not corrected by aids & $4 / 114(4)$ & $7 / 108(6)$ & 0.31 & $0.54(0.16-1.80)$ \\
\hline No useful vision & $8 / 119(7)$ & $12 / 114(11)$ & 0.30 & $0.64(0.27-1.50)$ \\
\hline $\begin{array}{l}\text { Seizures requiring anticonvulsant agents at } \\
\text { time of assessment }\end{array}$ & $12 / 116(10)$ & $16 / 116(14)$ & 0.42 & $0.75(0.37-1.51)$ \\
\hline $\begin{array}{l}\text { Head circumference at follow-up }>2 \text { SD } \\
\text { below the mean }\end{array}$ & $24 / 114(21)$ & $28 / 112(25)$ & 0.48 & $0.84(0.52-1.36)$ \\
\hline
\end{tabular}

* Scores on the Bayley Scales of Infant Development II (BSID-II) are assessed relative to a standardization mean ( \pm SD) of $100 \pm 15$, with higher scores indicating better performance. Scores on the Gross Motor Function Classification System (GMFCS) can range from 1 to 5, with higher scores indicating greater impairment. $\mathrm{Cl}$ denotes confidence interval.

come but had similar point estimates. The relative risk in the NICHD trial was 1.51 (95\% CI, 0.94 to 2.42$),{ }^{23}$ and the relative risk in the CoolCap trial was 1.48 (95\% CI, 0.89 to 2.45) (Gunn A, University of Auckland, New Zealand: personal communication).

Although there is striking homogeneity among results of these three trials, there are also some differences. Only the NICHD trial detected a significant effect of hypothermia on the primary outcome, and only TOBY detected significant improvements in specific neurologic outcomes. Both
TOBY and the CoolCap trial showed that the increased risk of death or severe disability in infants with the most abnormal grade on amplitudeintegrated electroencephalography was unaffected by cooling, but the CoolCap results suggested a reduction of the risk in the subgroup with less severely abnormal findings.

These discrepancies among results may be explained in part by differences in the trial protocols. In all three trials, the whole-body temperature (as measured in the rectum or esophagus) was reduced, but the strategies for cooling and 


\begin{tabular}{|c|c|c|c|c|}
\hline Outcome & $\begin{array}{l}\text { Cooled Group } \\
\quad(N=163)\end{array}$ & $\begin{array}{l}\text { Noncooled Group } \\
\qquad(\mathrm{N}=162)\end{array}$ & P Value & $\begin{array}{l}\text { Relative Risk or } \\
\text { Median Difference } \\
(95 \% \mathrm{Cl}) \dagger\end{array}$ \\
\hline \multicolumn{5}{|l|}{ Total duration of hospital care - days } \\
\hline Median & 12 & 13 & 0.13 & $1(0-4)$ \\
\hline IQR & $8-18$ & $9-25$ & & \\
\hline Persistent hypotension — no./total no. (\%) $\ddagger$ & $126 / 163(77)$ & $134 / 162(83)$ & 0.22 & $0.93(0.84-1.04)$ \\
\hline Prolonged coagulation time - no./total no. (\%) & $67 / 163(41)$ & $72 / 161(45)$ & 0.51 & $0.92(0.71-1.18)$ \\
\hline Thrombocytopenia — no./total no. (\%) & $94 / 163(58)$ & $80 / 161(50)$ & 0.15 & $1.16(0.95-1.42)$ \\
\hline Intracranial hemorrhage - no./total no. (\%) $\int$ & $25 / 64(39)$ & $21 / 67(31)$ & 0.35 & $1.25(0.78-1.99)$ \\
\hline \multicolumn{5}{|l|}{ Pulmonary diagnoses — no./total no. (\%) } \\
\hline Pneumonia & $5 / 163(3)$ & $5 / 162(3)$ & 0.99 & $0.99(0.29-3.37)$ \\
\hline Pulmonary air leak & $9 / 163(6)$ & $3 / 162(2)$ & 0.08 & $2.98(0.82-10.80)$ \\
\hline Pulmonary hemorrhage & $5 / 163(3)$ & $3 / 162(2)$ & 0.48 & $1.66(0.40-6.82)$ \\
\hline Pulmonary hypertension & $16 / 163(10)$ & $9 / 162(6)$ & 0.15 & $1.77(0.80-3.88)$ \\
\hline Necrotizing enterocolitis - no./total no. (\%) & $1 / 163(<1)$ & $0 / 162$ & & \\
\hline Cardiac arrhythmia — no./total no. (\%) $\rrbracket$ & $8 / 163(5)$ & $3 / 162(2)$ & 0.13 & $2.65(0.72-9.81)$ \\
\hline Culture-proven sepsis — no./total no. (\%) & $20 / 163(12)$ & $20 / 162(12)$ & 0.98 & $0.99(0.56-1.78)$ \\
\hline
\end{tabular}

* No case of renal failure requiring dialysis occurred. $\mathrm{Cl}$ denotes confidence interval, and IQR interquartile range.

$\dagger$ Values are relative risks except for total duration of hospital care, for which the median difference is shown.

Hypotension was defined as a mean blood pressure of $40 \mathrm{~mm} \mathrm{Hg}$ or less.

$\int$ Intracranial hemorrhage was identified on magnetic resonance imaging (MRI); 39 of the 46 cases were subdural (10 moderate and 29 mild). No intracranial hemorrhage was identified on cranial ultrasonography. Two cases of sinus thrombosis in the cooling group, and one case in the noncooled group, were also noted on MRI.

\All but three cases of cardiac arrhythmia consisted of sinus bradycardia of less than 80 beats per minute. The other three cases consisted of ventricular arrhythmia; two of these occurred in the noncooled group.

the target temperatures varied: temperature was decreased to 33 to $34^{\circ} \mathrm{C}$ with the use of cooling blankets in TOBY and the NICHD trial and to 34 to $35^{\circ} \mathrm{C}$ by means of scalp cooling in the CoolCap study. The NICHD trial recorded slightly higher temperatures in the control group than did the other two trials. In TOBY, but not the other two trials, cooling was initiated during transport to the treatment center. In TOBY and the CoolCap trial, but not the NICHD trial, patients were selected on the basis of the presence of abnormalities on amplitude-integrated electroencephalography in addition to clinical criteria. Differences in local practices for withdrawal of care may also have affected outcomes. Withdrawal was slightly more common in the control group than in the cooled group in the NICHD study but was more common in the cooled group than in the control group in TOBY; these results may partially account for the greater apparent effect of hypothermia on mortality rate in the NICHD study as compared with TOBY.

Elevation of body temperature to greater than $38^{\circ} \mathrm{C}$ was observed in several noncooled infants in the NICHD and CoolCap trials and was associated with a worse outcome in the CoolCap trial. ${ }^{11,24}$ A rectal temperature of more than $38^{\circ} \mathrm{C}$ was also noted in some noncooled infants in TOBY. Experimental data showing that pyrexia may adversely affect neurodevelopment support the possibility that increased temperatures may contribute to the poorer outcomes seen in the noncooled groups; however, it is also possible that the relationship between higher elevation of body temperature and poor outcome reflects reverse causation (i.e., asphyxia resulting in impairment of temperature regulation).

Consistent with findings in the earlier trials, in our study, minor respiratory and cardiovascular events were common, but serious adverse events 
were rare and were not associated with cooling. Mild-to-moderate intracranial hemorrhage that was not visible on cranial ultrasonography was frequently seen on MRI in both groups, and sinus thrombosis occurred very infrequently in both groups.

No trial, to our knowledge, has yet reported neurologic outcomes at ages older than 18 months. Neurodevelopmental assessments at 18 months may not reliably predict later outcomes. ${ }^{25}$ Although it is likely that severe neuromotor disability will have been correctly identified at 18 months, less severe impairments are not reliably assessable at this age. Assessment later in childhood (e.g., at 6 to 7 years of age) is necessary for accurate, comprehensive evaluation of cognitive function, be- havior and learning, fine motor development, attention, and psychosocial health. ${ }^{26}$

In conclusion, TOBY did not show a significant reduction in the combined rates of death and severe disability with cooling, as compared with no cooling, but did show a significant improvement in several secondary neurologic outcomes among survivors. Whether this improvement is maintained in the longer term needs to be ascertained.

Supported by grants from the U.K. Medical Research Council and the U.K. Department of Health.

No potential conflict of interest relevant to this article was reported.

We thank the Imperial College Healthcare Biomedical Research Centre and Bliss, the Special Care Baby Charity, for their advice and support, as well as all the parents and infants who took part in the study.

\section{APPENDIX}

Members of the TOBY Study Group are as follows: Project Management Group - D. Azzopardi (chief investigator), P. Brocklehurst (chief investigator), A.D. Edwards (principal investigator), H. Halliday (principal investigator), M. Levene (principal investigator), M. Thoresen (principal investigator), A. Whitelaw (principal investigator), S. Ayers (National Perinatal Epidemiology Unit [NPEU] information technology coordinator), U. Bowler (NPEU Clinical Trials Unit [CTU] senior trials manager), M. Gallagher (NPEU data manager), E. Juszczak (NPEU CTU head of trials), C. Mulhall (NPEU TOBY study coordinator), B. Strohm (NPEU TOBY research nurse and study coordinator); Writing Committee - D. Azzopardi (chief investigator), P. Brocklehurst (chief investigator), A.D. Edwards (principal investigator), E. Juszczak (NPEU CTU head of trials); Trial Steering Committee - N. McIntosh (chair), Child Life and Health, University of Edinburgh, Edinburgh, United Kingdom (UK); D. Azzopardi, Imperial College London, London; H. Baumer, Derriford Hospital, Plymouth, UK; P. Brocklehurst, NPEU, University of Oxford, Oxford, UK; C. Doré, Medical Research Council (MRC) CTU; D. Elbourne, London School of Hygiene and Tropical Medicine, London; R. Parnell, Scope Data Monitoring and Ethics Committee; R. Cooke (chair), Liverpool Women's Hospital, University of Liverpool, Liverpool, UK; H. Davies, National Research Ethics Service; A. Johnson, University of Oxford, Oxford, UK; S. Richmond, Sunderland District General Hospital, University of Newcastle, Newcastle, UK; P. Yudkin, Division of Public Health and Primary Health Care, University of Oxford, Oxford, UK; Trial Statisticians (NPEU) - S. Gates, Edmund Juszczak, M. Quigley; Trial Health Economists (NPEU) - O. Eddama, J. Henderson, S. Petrou; Clinical Research Fellow — O. Kapellou; Follow-up Pediatricians — L. Dyet, E. Porter, Imperial College London, London; G. Mero, Jósa András County Hospital, Nyíregyháza, Hungary; B. Vollmer, Karolinska Institutet, Stockholm; E. Goldstein, Soroka Medical Center, Beersheeva, Israel; Specialist Adviser - B. Hutchon, Royal Free Hospital, London; Cranial Ultrasonography Interpretation - C. Hagmann, University College London, London; Bayley Scales of Infant Development II (BSID-II) Training — S. Johnson, University of Nottingham, Nottingham, UK; MRI Evaluation - L. Ramenghi, University of Milan, Milan; M. Rutherford, MRC Clinical Sciences Centre, Imperial College London, London; Centers for Recruitment and Data Collection for MRI Evaluation (in descending order of no. of infants recruited, in parentheses) - Hammersmith Hospital, London (54) - D. Azzopardi, A.D. Edwards, O. Kapellou, P. Corcoran; First Department of Pediatrics, Semmelweis University Hospital, Budapest, Hungary (24) - M. Szabó, A. Róka, E. Bodrogi; Homerton Hospital, London (20) - E. Maalouf, C. Harris; Southmead Hospital, Bristol, UK (20) - A. Whitelaw, S. Lamburne; University College Hospital, London (19) — N. Robertson, A. Kapetanakis; St. George's Hospital, London (18) - K. Farrer, L. Kay-Smith; Royal Maternity Hospital, Belfast, UK (17) — H. Halliday, D. Sweet; Liverpool Women's Hospital, Liverpool, UK (15) - M. Weindling, A.S. Burke; St. Michael's Hospital, Bristol, UK (14) - M. Thoresen, J. Tooley, J. Kemp; Leicester Royal Infirmary, Leicester, UK (12) - A. Currie, M. Hubbard; Royal Sussex County Hospital, Brighton, UK (10) - P. Amess; Queen Silvia's Hospital, Gothenburg, Sweden (9) — K. Thiringer, A. Flisberg; Leeds General Infirmary, Leeds, UK (8) - M. Levene, A. Harrop; Nottingham City Hospital, Nottingham, UK (8) — S. Watkin, D. Jayasinghe; John Radcliffe Hospital, Oxford, UK (7) - E. Adams; Karolinska Institutet, Stockholm (6) - C. Lothian, M. Blennow; Medway Maritime Hospital, Gillingham, UK (6) - S. Rahman, B. Jani, K. Vandertak; Luton and Dunstable Hospital, Luton, UK (5) — S. Skinner, Y. Millar; Queen's Medical Centre, Nottingham, UK (5) - N. Marlow, S. Wardle; Jessop Wing, Sheffield, UK (4) - M. Smith; Royal Victoria Infirmary, Newcastle, UK (4) - J. Berrington; Soroka Medical Center, Beersheva, Israel (4) - K. Marks; Bradford Royal Infirmary, Bradford, UK (3) - S. Chatfield; Heartlands Hospital, Birmingham, UK (3) - S. Rose; New Cross Hospital, Wolverhampton, UK (3) - B. Kumararatne, L. Greig; Norfolk and Norwich University Hospital, Norwich, UK (3) - P. Clarke; Lund University Hospital, Lund, Sweden (3) - V. Fellman; Wishaw General Hospital, Wishaw, UK (3) - R. Abara; City Hospital, Birmingham, UK (2) — D. Armstrong; Erinville Hospital-Cork University Hospital, Cork, Ireland (2) - D. Murray; Hospital for Children and Adolescents, Helsinki (2) - M. Metsaranta; Queen Mother's Hospital, Glasgow, UK (2) - J. Simpson; Singleton Hospital, Swansea, UK (2) — J. Matthes; Southern General Hospital, Glasgow, UK (2) - P. MacDonald; University Hospital of Wales, Cardiff, UK (2) - S. Cherian; Princess Royal Maternity Hospital, Glasgow, UK (1) - L. Jackson; Royal Cornwall Hospital, Truro, UK (1) - P. Munyard; Royal Devon and Exeter Foundation Trust, Exeter, UK (1) - M. Quinn; St. Mary's Hospital, Manchester, UK (1) - S. Mitchell; James Cook University Hospital, Middlesbrough, UK (0) — S. Sinha; Derriford Hospital, Plymouth, UK (0) — J. Eason; Department of Pediatrics, Oulu University Hospital, Oulu, Finland (0) - M. Hallman. 
1. Thoresen M, Penrice J, Lorek A, et al. Mild hypothermia after severe transient hypoxia-ischemia ameliorates delayed cerebral energy failure in the newborn piglet. Pediatr Res 1995;37:667-70.

2. Sirimanne ES, Blumberg RM, Bossano $\mathrm{D}$, et al. The effect of prolonged modification of cerebral temperature on outcome after hypoxic-ischemic brain injury in the infant rat. Pediatr Res 1996;39:591-7.

3. Amess PN, Penrice J, Cady EB, et al. Mild hypothermia after severe transient hypoxia-ischemia reduces the delayed rise in cerebral lactate in the newborn piglet. Pediatr Res 1997;41:803-8.

4. Edwards AD, Yue X, Squier MV, et al. Specific inhibition of apoptosis after cerebral hypoxia-ischaemia by moderate postinsult hypothermia. Biochem Biophys Res Commun 1995;217:1193-9.

5. Bona E, Hagberg H, Loberg EM, Bågenholm R, Thoresen M. Protective effects of moderate hypothermia after neonatal hypoxia-ischemia: short- and long-term outcome. Pediatr Res 1998;43:738-45.

6. Colbourne F, Corbett D, Zhao Z, Yang J, Buchan AM. Prolonged but delayed postischemic hypothermia: a long-term outcome study in the rat middle cerebral artery occlusion model. J Cereb Blood Flow Metab 2000;20:1702-8.

7. Gunn AJ, Gluckman PD, Gunn TR. Selective head cooling in newborn infants after perinatal asphyxia: a safety study. Pediatrics 1998;102:885-92.

8. Azzopardi D, Robertson NJ, Cowan FM, Rutherford MA, Rampling M, Edwards AD. Pilot study of treatment with whole body hypothermia for neonatal encephalopathy. Pediatrics 2000;106:684-94.

9. Eicher DJ, Wagner CL, Katikaneni LP, et al. Moderate hypothermia in neonatal encephalopathy: safety outcomes. Pediatr Neurol 2005;32:18-24.

10. Gluckman PD, Wyatt JS, Azzopardi D et al. Selective head cooling with mild systemic hypothermia after neonatal encephalopathy: multicentre randomised trial. Lancet 2005;365:663-70.

11. Shankaran S, Laptook AR, Ehrenkranz RA, et al. Whole-body hypothermia for neonates with hypoxic-ischemic encephalopathy. N Engl J Med 2005;353:1574-84. 12. Shah PS, Ohlsson A, Perlman M. Hypothermia to treat neonatal hypoxic ischemic encephalopathy: systematic review. Arch Pediatr Adolesc Med 2007;161:951-8.

13. Schulzke SM, Rao S, Patole SK. A systematic review of cooling for neuroprotection in neonates with hypoxic ischemic encephalopathy - are we there yet? BMC Pediatr 2007;7:30.

14. Jacobs S, Hunt R, Tarnow-Mordi W, Inder T, Davis P. Cooling for newborns with hypoxic ischaemic encephalopathy. Cochrane Database Syst Rev 2007;4: CD003311.

15. Higgins RD, Raju TN, Perlman J, et al. Hypothermia and perinatal asphyxia: executive summary of the National Institute of Child Health and Human Development workshop. J Pediatr 2006;148:170-5.

16. Barks JD. Current controversies in hypothermic neuroprotection. Semin Fetal Neonatal Med 2008;13:30-4.

17. Kirpalani $\mathrm{H}$, Barks J, Thorlund $\mathrm{K}$, Guyatt G. Cooling for neonatal hypoxic ischemic encephalopathy: do we have the answer? Pediatrics 2007;120:1126-30.

18. al Naqeeb N, Edwards AD, Cowan FM, Azzopardi D. Assessment of neonatal encephalopathy by amplitude-integrated elec- troencephalography. Pediatrics 1999;103: 1263-71.

19. Allmark P, Mason S. Improving the quality of consent to randomised controlled trials by using continuous consent and clinician training in the consent process. J Med Ethics 2006;32:439-43.

20. Palisano RJ, Hanna SE, Rosenbaum PL, et al. Validation of a model of gross motor function for children with cerebral palsy. Phys Ther 2000;80:974-85.

21. Haataja L, Mercuri E, Regev R, et al. Optimality score for the neurologic examination of the infant at 12 and 18 months of age. J Pediatr 1999;135:153-61.

22. Bayley N. Bayley scales of infant development. 2nd ed. San Antonio, TX: Psychological Corporation, 1993.

23. Shankaran S, Pappas A, Laptook AR, et al. Outcomes of safety and effectiveness in a multicenter randomized, controlled trial of whole-body hypothermia for neonatal hypoxic-ischemic encephalopathy. Pediatrics 2008;122(4):e791-e798. 24. Wyatt JS, Gluckman PD, Liu PY, et al. Determinants of outcomes after head cooling for neonatal encephalopathy. Pediatrics 2007;119:912-21.

25. Barnett AL, Guzzetta A, Mercuri E, et al. Can the Griffiths scales predict neuromotor and perceptual-motor impairment in term infants with neonatal encephalopathy? Arch Dis Child 2004;89:637-43. 26. Voss W, Neubauer AP, Wachtendorf M, Verhey JF, Kattner E. Neurodevelopmental outcome in extremely low birth weight infants: what is the minimum age for reliable developmental prognosis? Acta Paediatr 2007;96:342-7.

Copyright (๐ 2009 Massachusetts Medical Society.

POWERPOINT SLIDES OF JOURNAL FIGURES AND TABLES At the Journal's Web site, subscribers can automatically create PowerPoint slides. In a figure or table in the full-text version of any article at NEJM.org, click on Get PowerPoint Slide. A PowerPoint slide containing the image, with its title and reference citation, can then be downloaded and saved. 